\title{
The twists and turns of rotating turbulence
}

\author{
STUART B. DALZIEL \\ Department of Applied Mathematics and Theoretical Physics, \\ University of Cambridge, Wilberforce Road, \\ Cambridge CB3 0WA, UK
}

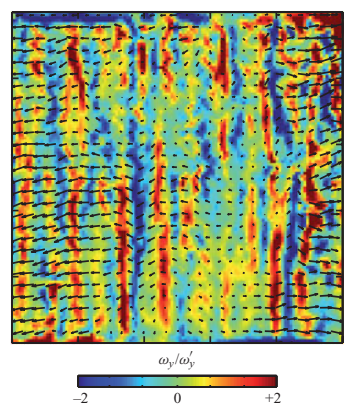

Turbulence is widely considered one of the most important and most difficult unsolved problems in classical physics. It is also the area of fluid mechanics where the greatest effort is exerted, the most papers published and, some would argue, the least progress made. Although direct numerical simulation is becoming an increasingly valuable tool, there remains a need for high-quality experiments to underpin our theoretical and numerical progress. Such statements apply equally to the 'classical' problem of homogeneous isotropic turbulence and to turbulence in its many other guises. Of particular interest is turbulence in a rotating system, where it is well known that the influence of rotation leads to the development of anisotropy and the elongation of scales parallel to the rotation axis. Moisy et al. (J. Fluid Mech., 2010, this issue, vol. 666, pp. 5-35) present new experiments in the free decay of grid-generated turbulence in a rotating system. They investigate the emergence of anisotropy from essentially isotropic initial conditions. While it is well known that rotation suppresses velocity gradients parallel to the rotation axis, Moisy et al. (2010) uncover some startling and previously overlooked implications.

Key words: rotating turbulence

\section{Introduction}

Much of our progress in understanding turbulence has been made through study of the artificial problem of homogeneous, isotropic turbulence. Even this 'simple' case defies much beyond the well-known and often abused Kolmogorov $k^{-5 / 3}$ energy spectrum for the inertial range. (Here $k$ is the wavenumber.) Add in rotation (assumed here to be about a vertical axis) and things change. Whereas decaying grid-generated turbulence in a non-rotating system remains approximately isotropic (figure $1 a$ ), the vertical length scale increases dramatically in the rotating case (figure $1 b$ ). This anisotropy is not due to choosing a different reference frame, but rather due to changing the state to which the turbulence decays from one of rest to one of solidbody rotation in an inertial frame.

For a turbulent flow, the relative importance of inertia and the Coriolis force, due to rotation with angular velocity $\Omega$, can be described by the turbulent Rossby number,

$$
R o=\frac{u^{\prime}}{2 \Omega L} .
$$


(a)

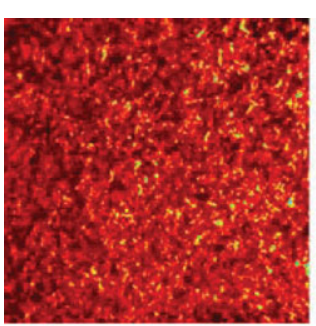

(b)

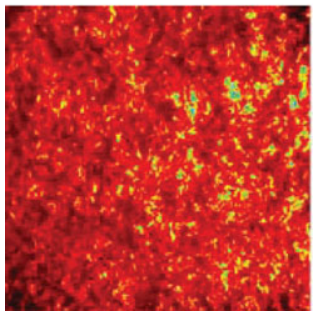

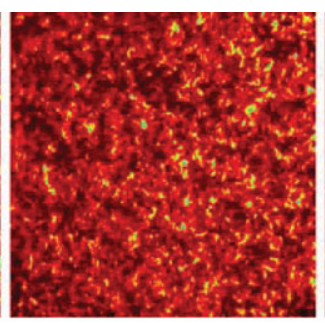

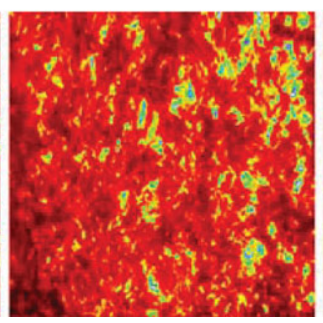

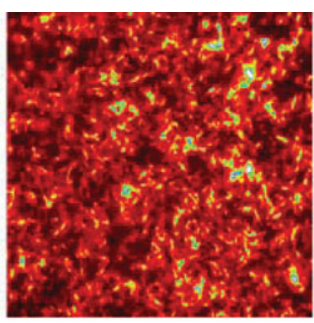
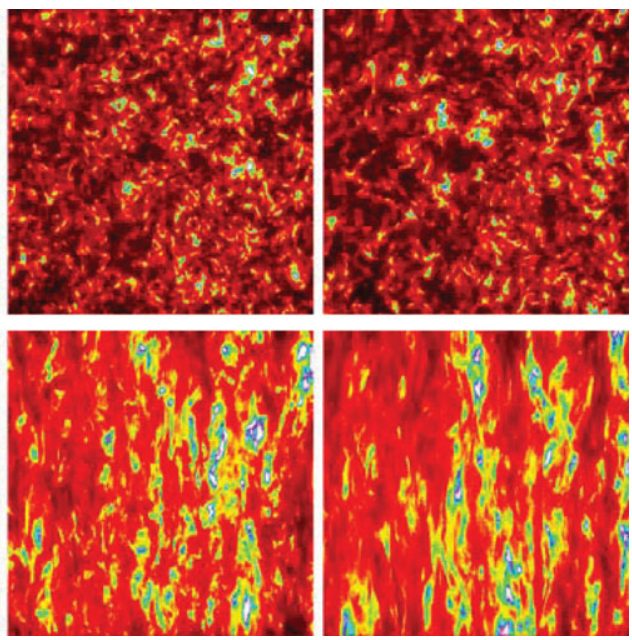

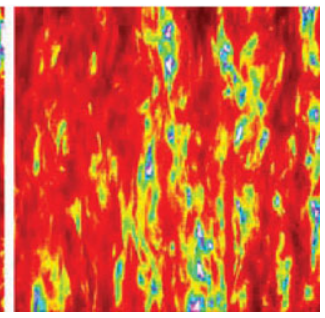

FIGURE 1. The emergence of anisotropy. Pearlescence images of decaying turbulence behind a downward propagating grid in non-rotating $(a)$ and rotating $(b)$ experiments. Images shown at $U t / M \approx 10,20,40,80(\Omega t / 2 \pi \approx 1 / 2,1,2,4)$ after the grid left the bottom of the field of view. (Images courtesy P. J. Staplehurst.)

If initially $R o \gg 1$, then rotation plays little role, but inevitably the decay of the turbulence intensity $u^{\prime}$ and increase in the integral length scale $L$ will reduce the Rossby number to the point where both inertia and rotation are important and anisotropy emerges from isotropic initial conditions. As Ro continues to decrease, the motion becomes dominated by inertial waves of ever reducing frequencies, with the flow becoming increasingly two-dimensional as vertical velocity gradients decrease.

Our understanding of rotating turbulence has progressed alongside but lagged a little behind its non-rotating counterpart, with experiments playing a defining role. Although they were not the first to perform rotating experiments, Ibbetson \& Tritton (1975) were the first to provide hard data. Their turbulence was generated in air by two perforated plates moving apart in a direction parallel to the rotation axis in a rotating annular geometry, and measured by 'flying' hot-wire probes around the annulus on a streamlined rotating arm. Their measurements not only confirmed the expected development of anisotropy with an increase in the vertical length scales, but also suggested that the relative strength of the horizontal and vertical velocity fluctuations did not change dramatically. This second point is now of great interest. Ibbetson and Tritton also observed that the decay rate increased with the rotation rate, a feature they were suspicious of and attributed to the low aspect ratio of their domain: it is now widely accepted (e.g. Jacquin et al. 1990) that rotation actually decreases the decay rate through the removal of the vortex stretching mechanism that helps take energy to smaller scales.

\section{Overview}

Skipping over many insightful contributions presented in the intervening 35 years, we turn to the study by Moisy et al. (2010). Technology has moved on, allowing much 
more detailed measurements. Strapping a high-resolution particle image velocimetry system to a rotating table is now relatively easy, but the manner in which the results are used remains as challenging as ever. While this very careful study is a development of earlier work, it is much more than just the next paper in a series. Even the scale of the experiments, using the $14 \mathrm{~m}$ diameter Coriolis rotating table in Grenoble, places this study of grid-generated turbulence in a league of its own.

How does the anisotropy emerge? With the initial Rossby number high enough for the rotation not to be important, the initial decay sees the velocity fluctuations decrease as $u^{\prime 2} \sim t^{-6 / 5}$ and the integral length scale increases as $L \sim t^{2 / 5}$. Remarkably, combining these into the turbulent Rossby number shows that $R o \sim(\Omega t)^{-1}$, where the prefactor is independent of the grid velocity or mesh size. The experiments show that anisotropy emerges quite suddenly as $R o$ decreases through 0.25 and that this occurs around 0.4 of a rotation period after passage of the grid.

The change in behaviour for $R o<0.25$ can be seen in a number of ways. The decay rate of both horizontal and vertical velocity fluctuations decreases from $u^{\prime 2} \sim t^{-6 / 5}$ to something consistent with $t^{-3 / 5}$, while the integral length scale (constructed from horizontal velocities separated in the horizontal direction) continues to follow the same $L \sim t^{2 / 5}$ scaling seen initially. The vertical correlation of horizontal velocities, however, increases rapidly, reflecting the suppression of $\partial u / \partial z$ and $\partial v / \partial z$, leaving behind a dominance of cyclones over anticyclones.

Despite this two-dimensionalisation of the flow, the ratio of the vertical to horizontal velocity fluctuations remains approximately constant, in line with the observations of Ibbetson \& Tritton (1975). More surprisingly, the vertical correlation of the vertical velocity only grows a little faster than the horizontal correlation of the horizontal velocity, despite the suppression of $\partial w / \partial z$. What Moisy et al. (2010) suggest happens to the vertical velocity fluctuations is one of the major surprises.

To understand this better, it helps to think about the limit of two-dimensional turbulence. In this limit, there is no vortex stretching and vertical vorticity (normal to the plane of the motion) obeys the advection-diffusion equation as though it were a passive scalar. The spectral relationship $\Phi(k)=k^{2} E(k)$ between energy density $E(k)$ and enstrophy (mean square vorticity) density means that since neither $E(k)$ nor $\Phi(k)$ can increase due to diffusion, any spectral transfer of energy to higher wavenumbers must be balanced by a greater transfer of energy to lower wavenumbers to avoid $\Phi(k)$ increasing. This so-called anticascade of energy, whereby most of the energy moves to increasingly larger scales as the flow decays, occurs alongside a cascade of enstrophy to smaller scales.

In decaying rotating turbulence, the flow becomes quasi-two-dimensional at large scales as the Rossby number decreases, allowing an anticascade of energy and cascade of enstrophy to develop. The vertical velocity, which is organised in vertical sheets and does not play an active role in the equations in the low $R o$ quasi-two-dimensional limit, obeys the same advection-diffusion equation as the vertical vorticity. Like the enstrophy, the vertical velocity fluctuations are cascaded towards smaller horizontal scales. Moisy et al. suggest that vertical velocity $w$ present before the anisotropy develops continues to decay gradually due to molecular diffusion, but the horizontal gradients $\partial w / \partial x$ and $\partial w / \partial y$ can increase due to the cascade. Unlike a passive scalar, this increase in gradient leads at later time to shear instabilities between sheets of fluid moving vertically. Whereas the large-scale flow may be quasi-two-dimensional, these shear instabilities are not, providing a re-injection of three-dimensional motion (and small-scale vertical vorticity of both signs) at later time. 


\section{Future}

High-quality data like these will help fuel the current resurgence of interest in rotating turbulence. However, we must always be aware of the truth in the adage that everyone believes experimental results except the experimentalist. There are imperfections despite the considerable care and skill of the authors. The channel used had a depth of only seven times the mesh spacing, which raises questions about the influence of confinement. Confinement also plays a role in the systematic flow generated by the passage of the grid (Dalziel 1992) and how this couples with the turbulence in a non-trivial way. While Moisy et al. have made a Reynolds decomposition to remove the leading-order effect from their analysis, the validity of this approach at later times is unknown. It may yet prove necessary to explicitly include this component in a numerical model in order to match all details of the evolution, or to wait for the next generation of experiments where the imperfections are reduced, or at least different.

The experiments explore the transition through $R o=O(1)$, where both nonlinear and linear effects must be important. However, while the drive towards anisotropy is clear and the form taken has been characterised, details of the manner in which this is achieved remain unclear, as is the importance of an initial value of $R o$ that is itself only of $O(1)$. Moisy et al. (2010) steer clear of current debate between the dominance of nonlinear triadic interactions (e.g. Cambon, Mansour \& Godeferd 1997 and Smith \& Waleffe 1999) and the possible role of linear wave propagation (e.g. Staplehurst, Davidson \& Dalziel 2008 and Kolvin et al. 2009), yet their results will feed into the discussion.

While theoreticians will use these data to inspire new approaches and numerical modellers will try to replicate the results, experimentalists will dream up new ways of providing even better data. These results are based on two-dimensional measurements of the velocity field in two planes - one horizontal and the other vertical in separate experiments - yet three-dimensional technologies are available. However, it may take some time before the benchmark set here is superseded, particularly with the Coriolis table having to be moved to make way for a new roadway.

\section{References}

Cambon, C., Mansour, N. N. \& Godeferd, F. S. 1997 Energy transfer in rotating turbulence. J. Fluid Mech. 337, 303-332.

Dalziel, S. B. 1992 Decay of rotating turbulence: some particle tracking experiments. Appl. Sci. Res. 49, 217-244.

Ibbetson, A. \& Tritton, D. J. 1975 Experiments on turbulence in a rotating fluid. J. Fluid Mech. 68, 639-672.

Jacquin, L., Leuchter, O., Cambon, C. \& Mathieu, J. 1990 Homogeneous turbulence in the presence of rotation. J. Fluid Mech. 220, 1-52.

Kolvin, I., Cohen, K., Vardi, Y. \& Sharon, E. 2009 Energy transfer by inertial waves during the buildup of turbulence in a rotating system. Phys. Rev. Lett. 102, 014503.

Moisy, F., Morize, C., Rabaud, M. \& Sommeria, J. 2010 Decay laws, anisotropy and cycloneanticyclone asymmetry in decaying rotation turbulence. J. Fluid Mech. 666, 5-35.

Smith, L. M. \& WalefFe, F. 1999 Transfer of energy to two-dimensional large scales in forced, rotating three-dimensional turbulence. Phys. Fluids 11, 1608-1622.

Staplehurst, P. J., Davidson, P. A. \& Dalziel, S. B. 2008 Structure formation in homogeneous freely-decaying, rotating turbulence. J. Fluid Mech. 598, 81-105. 